ఠ

REVIEW

\title{
A case of hypertrophic olivary degeneration after resection of cavernomas of the brain stem and review of the literature
}

This article was published in the following Dove Press journal:

Neuropsychiatric Disease and Treatment

8 October 2015

Number of times this article has been viewed

\section{Meng Zhang \\ Gengfan Ye \\ Lin Deng \\ Shuo Xu \\ Yunyan Wang}

Department of Neurosurgery, Qi Lu Hospital, Shandong University, Jinan, People's Republic of China
Correspondence: Yunyan Wang Department of Neurosurgery, Qi Lu Hospital, Shandong University, I07 Wenhua Xi Road, Jinan 2500 I2, People's Republic of China $\mathrm{Tel} / \mathrm{fax}+86$ 53I 8216 66I5 Email wangyyan0618@yeah.net

\begin{abstract}
Hypertrophic olivary degeneration is a transsynaptic form of degeneration, which is also a result of primary or secondary lesion and can damage the dento-rubro-olivary pathway. The dento-rubro-olivary pathway was first described by Guillain and Mollaret and is referred to as "the triangle of Guillain and Mollaret". Multiple factors can destroy the dento-rubro-olivary pathway, such as surgical operation, hemorrhage, tumor, trauma, inflammation, demyelination, degeneration, and radiation damage. All of the above factors can result in delayed hypertrophic olivary degeneration. Articles related to this disease cover etiology, clinical presentation, pathology changes, etc. However, to our knowledge, there has been no literature reporting the use of diffusion tensor imaging and diffusion tensor tractography to improve the diagnosis of hypertrophic olivary degeneration following resection of cavernomas in the brain stem. Herein, we report a case who was diagnosed with hypertrophic olivary degeneration following resection of cavernomas of the brain stem, verify the significance of diffusion tensor imaging and diffusion tensor tractography, and review previous literature. The development of imageology promotes and improves hypertrophic olivary degeneration diagnosis and differential diagnosis.
\end{abstract}

Keywords: HOD, diffusion tensor imaging, diffusion tensor tractography

\section{Introduction}

Hypertrophic olivary degeneration (HOD) is an unusual neuronal degenerative disease involving inferior olivary nuclei with increased T2-weighted signal intensity on magnetic resonance imaging (MRI). Oppenheim first described HOD in 1887. ${ }^{1,2}$ However, Guillain and Mollaret widely described and studied the HOD with more detailed experiments in 1931 and first reported the theory of the dento-rubro-olivary pathway, which is referred to as "the triangle of Guillain and Mollaret".,3 Researchers have reported that HOD is a transsynaptic disease of degeneration and that the inferior olivary nucleus is hypertrophic, but not atrophic. It has also been reported that many factors can affect the dento-rubro-olivary pathway, such as surgical operation, hemorrhage, tumor, trauma, inflammation, demyelination, degeneration, and radiation damage. ${ }^{5-8}$ In a recent study, we can see the etiology, clinical manifestation, MRI presentation, and prognosis of HOD clarified in detail. ${ }^{9}$ Although the theory of the triangle of Guillain and Mollaret has been described, diagnosing HOD following resection of cavernomas of the brain stem by using diffusion tensor imaging (DTI) and diffusion tensor tractography (DTT) has not been reported. Therefore, we report a case concerning delayed HOD after resection of cavernomas of the brain stem and demonstrate the value and significance of DTI and DTT in diagnosis of HOD. This article will present new knowledge of HOD by means of a case report and review of the literature. 


\section{Case report}

A 62-year-old woman was hospitalized in the Department of Neurosurgery in Qi Lu Hospital (Jinan, People's Republic of China). The chief complaint was sudden-onset dizziness and superficial sensory decrease in the right limbs for 1 week. The computed tomography scan results showed that there was a high-density change in the posterior cranial fossa, which was considered to be a hemorrhage of the pons. There was soft high signal intensity on T1-weighted imaging and high signal intensity on T2-weighted imaging (T2WI), with a low signal surrounding the lesion in the MRI scan. We considered the disease to be cavernous hemangioma or cavernoma (Figure 1). Then, we performed a cavernoma resection via posterior fossa craniotomy and confirmed the disease histologically. The patient recovered well and was discharged 10 days after operation without apparent abnormal signal on the primary lesion area on MRI (Figure 2). One year later, the patient was admitted to Qi Lu Hospital again, with diplopia, restricted motion of the left eye, involuntary movement of the uvula, and inaccuracy of bilateral finger-to-nose test and heel-knee-tibia test. MRI scan displayed an enlarged mass in the left anterolateral part of the medulla, with a high signal on T2WI and no obvious increased signal intensity on enhancement scan (Figure 3). The DTI and DTT showed the nerve fiber of the left was decreased compared to the right and the other part of the interrupted areas (Figure 4). We made the diagnosis of HOD and did follow-up visits at regular intervals.

We continue to perform clinical and radiologic (T2WI) follow-up, and the patient has shown HOD-associated symptoms on radiologic imaging at each time of follow-up. Therefore, we can confirm that our diagnosis of HOD is correct. Written, informed consent was obtained from the patient. This study has also been approved by the ethics committee of Shandong University, Jinan, People's Republic of China.

\section{Discussion}

HOD was first described by Oppenheim in $1887,{ }^{1,2}$ and HOD was chacterized by cytoplasmic vacuolation and the pathological changes of the neural cell body enlargement. ${ }^{10}$ Ogawa et $\mathrm{al}^{11}$ confirmed the clinical pathology by postmortem examination. According to a previous report about HOD, there were no significant differences in morbidity according to age, sex, and single or multiple lesions, but there were for position. That is to say, the morbidity of HOD was higher when the primary disease was located in the mesencephalon. ${ }^{12}$ Clinical manifestations of HOD include dentatorubral tremor, ocular myoclonus, diplopia, and palatal myoclonus. The high signal intensity on T2WI and absence of contrast enhancement could help us to distinguish HOD from a tumor lesion or an infection. ${ }^{13-15} \mathrm{~A}$ lesion-damaged dento-rubro-olivary pathway could result in HOD, according to Guillain and Mollaret's report in 1931.,4

Anatomically, the dento-rubro-olivary pathway, or triangle of Guillain and Mollaret, is composed of three structures, including the ipsilateral red nucleus in the midbrain, ipsilateral inferior olivary nucleus in the medulla, and contralateral dentate nucleus in the cerebellum. The efferent nerve tract travels from the dentate nucleus, ascends to the contralateral red nucleus via the superior cerebellar peduncle, and forms the decussation. Then, the tract descends through the central tegmental tract to the ipsilateral inferior olivary nucleus. Finally, the afferent pathway originates from the inferior olivary nucleus to the contralateral cerebellum bypassing the inferior cerebellar peduncle. The above clarifies the pathway of the triangle of Guillain and Mollaret. ${ }^{16}$ If a lesion damages the red nucleus or central tegmental tract, degeneration occurs in the ipsilateral inferior olivary nucleus. On the contrary, if the dentate nucleus or superior cerebellar peduncle is insulted, degeneration would
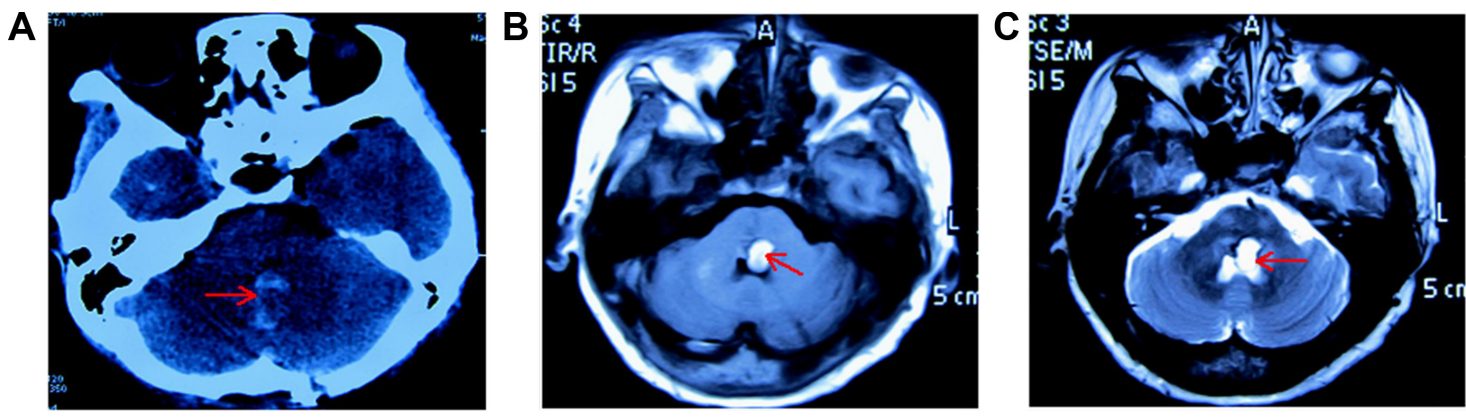

Figure I CT and MRI scan results in a case of hypertrophic olivary degeneration following resection of a cavernoma in the brain stem.

Notes: (A) CT scan showing cerebral hemorrhage in the posterior cranial fossa. High signal intensity on both TI-weighted imaging (B) and T2-weighted imaging (C), with low signal surrounding the lesion in the MRI scan. The arrows illustrate the lesion.

Abbreviations: CT, computed tomography; MRI, magnetic resonance imaging. 

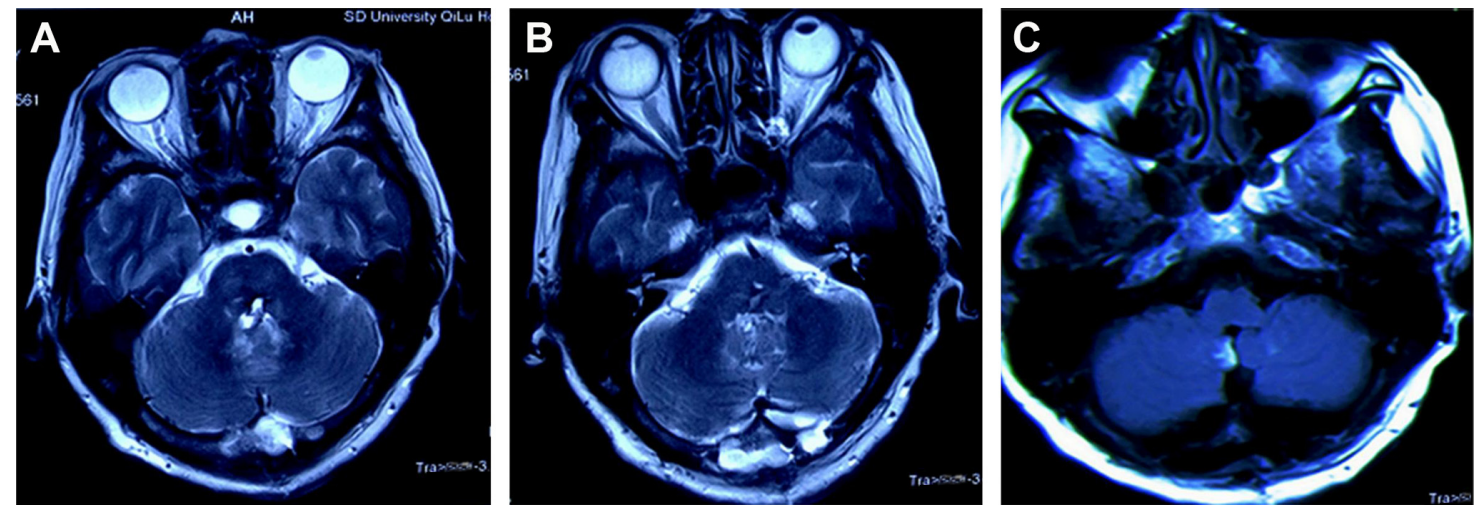

Figure 2 The surgical site without apparent abnormal signal in MRI.

Notes: (A) No abnormal signal intensity on TI-weighted imaging. (B) No abnormal signal intensity on T2-weighted imaging in the MRI scan. (C) Surgical spot without apparent abnormal signal.

Abbreviation: MRI, magnetic resonance imaging.

happen in the contralateral corresponding area. The morbidity of bilateral HOD is rather lower than that of unilateral lesion. Only if the pathological changes damage both central tegmental tracts and the bilateral superior cerebellar peduncle or the decussation of the superior cerebellar peduncle would bilateral HOD result. ${ }^{17} \mathrm{HOD}$ often happens when the dentate nucleus-red nucleus pathway or central tegmental tract pathway is damaged, rather than the pathway from the inferior olivary nucleus to the contralateral cerebellum. ${ }^{18}$

The clinical presentation of HOD includes oscillopsia diplopia and palatal myoclonus. ${ }^{19}$ To our knowledge, the inferior olivary nucleus could regulate the cerebellum
A

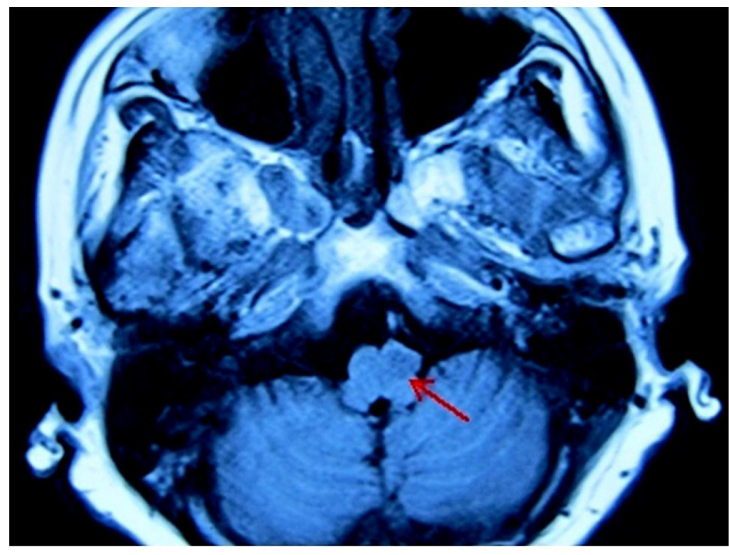

C

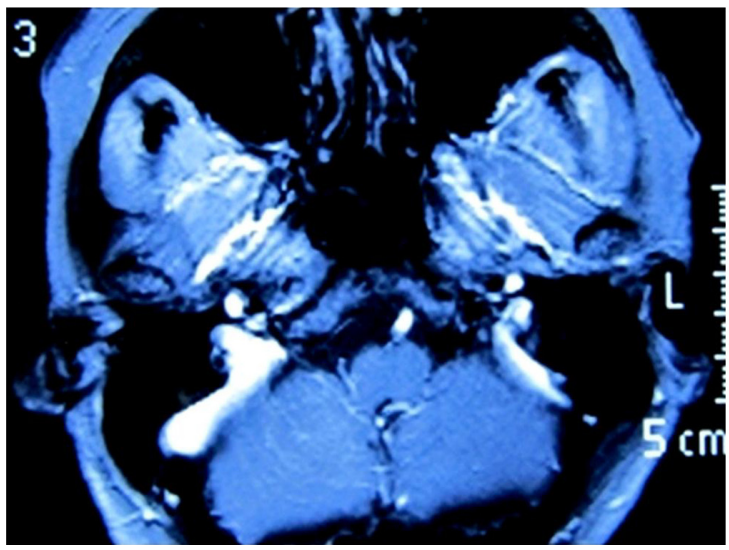

B
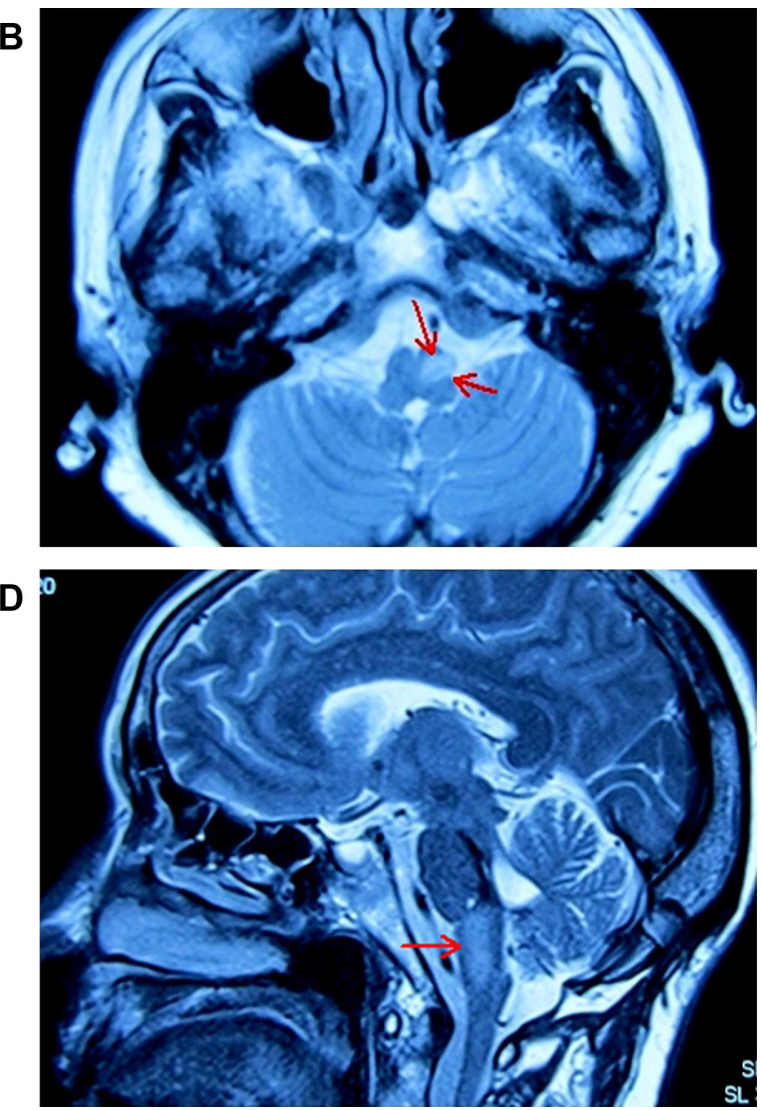

Figure 3 The signal intensity on TI-weighted imaging (TIWI) and T2-weighted imaging (T2WI) in the left anterolateral part of the medulla.

Notes: An enlarged mass in the left anterolateral part of the medulla, with low signal intensity on TIWI (A) and high signal intensity on T2WI (B) (arrows). (C) No obvious increased signal intensity on enhancement scan. (D) Sagittal imaging: high signal intensity on T2WI (arrow) in the medulla. 


\section{A}

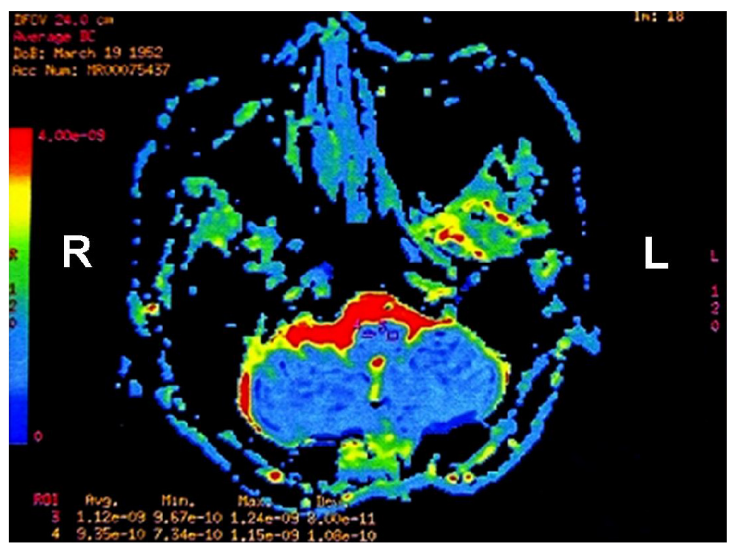

C

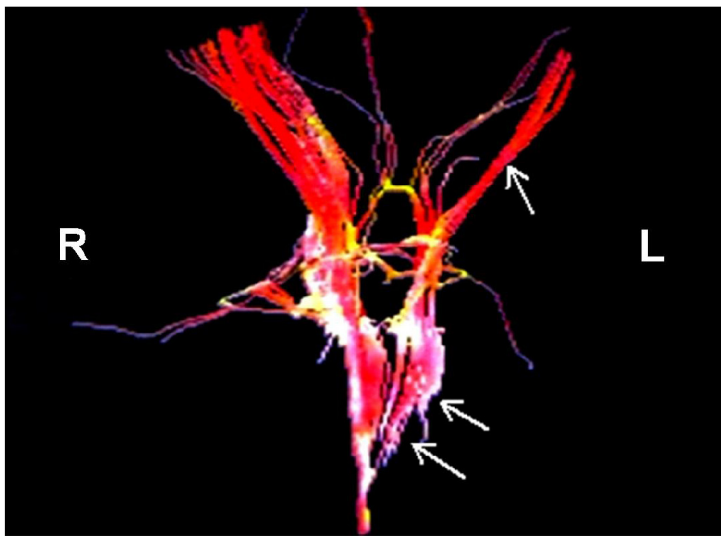

B

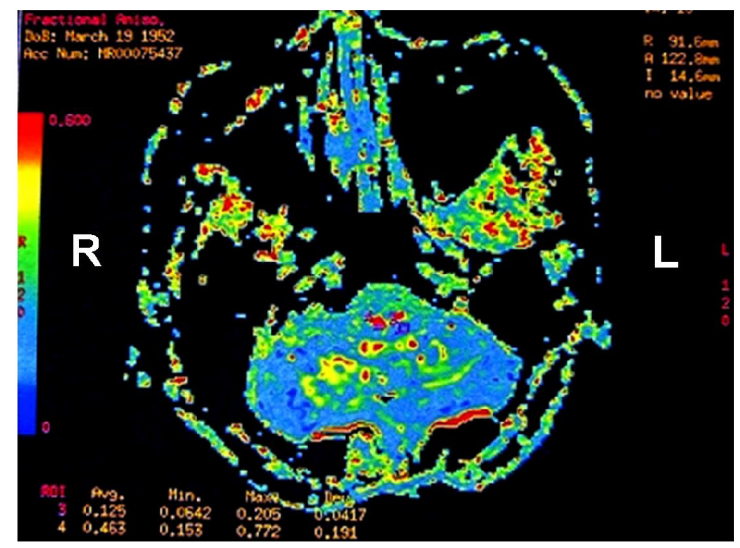

D

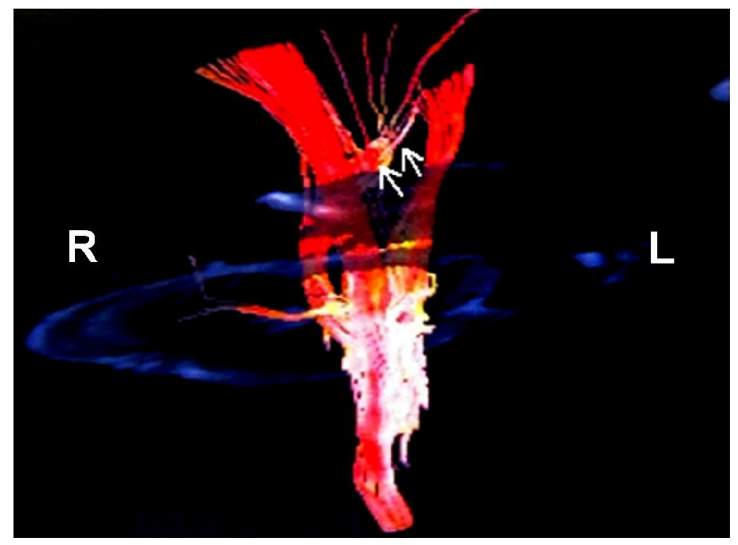

Figure 4 Diffusion tensor imaging ( $\mathbf{A}$ and $\mathbf{B}$ ) and diffusion tensor tractography ( $\mathbf{C}$ and $\mathbf{D})$ observation of the nerve fiber.

Notes: (A) The value of the apparent diffusion coefficient in the left is greater than that in the right. (B) The value of fractional anisotropy in the left is less than that in the right. (C) The nerve fiber tract (upper white arrow) of the left is decreased compared to the right and part of that interrupted (lower two white arrows). (D) The right superior cerebellar peduncle (white arrows) ascending from lower right to upper left; the left superior cerebellar peduncle is not visualized.

Abbreviations: L, left side; $R$, right side.

neural activity by generating an inhibitory substance, such as gamma-aminobutyric acid (GABA). ${ }^{17,20}$ When some pathological changes may have damaged the triangle of Guillain and Mollaret and clinical symptoms (such as palatal myoclonus) occur, the diagnosis of HOD should be strongly suspected. Nevertheless, not all patients of HOD will have palatal myoclonus.

The time interval between pathological changes and MRI manifestation of HOD can vary to some extent. Pathological changes of the inferior olivary nucleus can be classified into six stages: stage 1: no apparent changes of the inferior olivary nucleus in both macropathology and microscopic pathology; stage 2: degeneration of the olivary amiculum and inferior olivary nucleus neuron pathological hypertrophy with no glial proliferation under the microscope after approximately 3 weeks; stage 3: soft hypertrophy of the inferior olivary nucleus because of neuron and astrocyte proliferation after approximately 6 months; stage 4 : significant hypertrophy and enlargement of the inferior olivary nucleus; stage 5: olivary pseudohypertrophy, which persists for 3-4 years; and stage 6: inferior olivary nucleus atrophy due to neuronal disappearance. ${ }^{12,20,21}$ In addition, researchers have recently assessed the MRI presentation and propose three phases of HOD: phase 1: increased signal begins on T2WI in about the third week after lesion and without olivary changes within 6 months; phase 2: both olivary hypertrophy and high signal on T2WI, persisting for approximately 3-4 years; and phase 3: disappearance of olivary hypertrophy and persistence of increased signal on T2WI for a long time. ${ }^{20-23}$ The present case of HOD belongs to the second phase and corresponded to pathological changes and MRI manifestation. The HOD diagnosis was further verified by DTI and DTT.

DTI is a method which can analyze the diffusion movement of water molecules in human tissue in three-dimensional space on the basis of fractional anisotropy (FA) of water hydrone distribution. ${ }^{24}$ The parameter is described by FA value, which is related to the compactness and completeness of the neural fiber tract. By FA imaging and directionally encoded color imaging, the white matter fiber could be 
visualized by a special method to evaluate the completeness of the organic structure. ${ }^{25,26}$ The bilateral central tegmental tracts are distributed symmetrically and uniformly on DTT at the anatomical level, as is the decussation of the superior cerebellar peduncle. When a primary lesion damages the area of the triangle of Guillain and Mollaret, the DTT will be abnormal. From the present case of HOD, in Figure 4, we can see the value of the apparent diffusion coefficient increased significantly on the left diseased region compared to the right diseased region. Similarly, the value of FA decreased relative to the contralateral area. The DTI and DTT showed that the nerve fiber tract of left was decreased compared to the right and the other part of that interrupted. However, there was no apparent change in the decussation of the superior cerebellar peduncle, which could illustrate that the lesion mainly damaged the left central tegmental tract and the left superior cerebellar peduncle rather than the decussation. According to the theory of the triangle of Guillain and Mollaret, damage to the ipsilateral central tegmental tract could result in ipsilateral HOD. The present case was a patient with HOD after resection of cavernomas of the left dorsomedial pons, which can insult the left central tegmental tract and lead to HOD. The diagnosis of this case was made according to the presentation of DTT and DTI.

In clinical studies, HOD was mainly caused by two kinds of treatment, including surgical resection and gamma knife radiosurgery of brain stem cavernomas. ${ }^{27,28}$ The frequency of HOD caused by surgical resection was equal to that caused by gamma knife radiosurgery. ${ }^{27,28}$ Yun et $\mathrm{al}^{21}$ reported that the time interval from resection to HOD diagnosis was a mean of 7.7 months (range: 1-12 months) in surgical resection and 9.5 months (range: 3-15 months) in the gamma knife radiosurgery. Therefore, the interval between resection and HOD occurrence is random, and not limited within a certain period range.

\section{Conclusion}

HOD, as a rare disease of transsynaptic degeneration, has been the focus of an increasing amount of research in recent years. The development of imageology promotes and improves the level of HOD diagnosis and differential diagnosis. Unfortunately, knowledge of HOD is still so limited and many mechanics of this disease are still so unclear that the therapeutic outlook is fairly pessimistic. More research and effort need to be put into practice.

\section{Acknowledgments}

This work was supported by the National Natural Scientific Foundation of China (number 81141088) and by the Promotive Research Fund for Excellent Young and Middle-aged Scientists of Shandong Province (number 2004BS02010).

\section{Disclosure}

The authors report no conflicts of interest in this work.

\section{References}

1. Oppenheim H. Über Olivendegeneration bei Atheromatose der basalen Hirnarterien. Berl Klin Wochenschr. 1887;34:638-639. German.

2. Mancuso M, Orsucci D, Choub A, Siciliano G. Current and emerging treatment options in the management of Friedreich ataxia. Neuropsychiatr Dis Treat. 2010;6:491-499.

3. Masson M, Prier S, Henin D. [Post-anoxic intention and action myoclonies: neuropathological study in one case]. Rev Neurol (Paris). 1980;135:923-924. French.

4. Vaidhyanath R, Thomas A, Messios N. Bilateral hypertrophic olivary degeneration following surgical resection of a posterior fossa epidermoid cyst. Br J Radiol. 2010;83:e211-e215.

5. Santos AF, Rocha S, Varanda S, et al. Hypertrophic olivary degeneration and cerebrovascular disease: movement in a triangle. $J$ Stroke Cerebrovasc Dis. 2015;24:e59-e60.

6. Marden FA. Hypertrophic olivary degeneration due to pontine hemorrhage. JAMA Neurol. 2013;70:1330.

7. Menezes Cordeiro I, Tavares JB, Reimão S, Geraldes R, Ferro JM Hypertrophic olivary degeneration after pontine hemorrhage: a cause of delayed neurological deterioration. Cerebrovasc Dis. 2013;36: $153-154$.

8. Siebert E, Harms L, Herbst M. Posttraumatic bilateral hypertrophic olivary degeneration. Neurol Sci. 2013;34:1829-1830.

9. Carr CM, Hunt CH, Kaufmann TJ, Kotsenas AL, Krecke KN, Wood CP. Frequency of bilateral hypertrophic olivary degeneration in a large retrospective cohort. J Neuroimaging. 2015;25:289-295.

10. Otto J, Guenther P, Hoffmann KT. Bilateral hypertrophic olivary degeneration in Wilson disease. Korean J Radiol. 2013;14:316-320.

11. Ogawa K, Mizutani T, Uehara K, Minami M, Suzuki Y, Uchihara T. Pathological study of pseudohypertrophy of the inferior olivary nucleus. Neuropathology. 2010;30:15-23.

12. Pandey P, Westbroek EM, Gooderham PA, Steinberg GK. Cavernous malformation of brainstem, thalamus, and basal ganglia: a series of 176 patients. Neurosurgery. 2013;72:573-589.

13. Bruno MK, Wooten GF. Hypertrophic olivary degeneration. Arch Neurol. 2012;69:274-275.

14. Crosbie I, McNally S, Brennan P, Looby S. Teaching NeuroImages: hemorrhagic cavernoma with secondary development of hypertrophic olivary degeneration. Neurology. 2013;80:e199-e200.

15. Hornyak M, Osborn AG, Couldwell WT. Hypertrophic olivary degeneration after surgical removal of cavernous malformations of the brain stem: report of four cases and review of the literature. Acta Neurochir (Wien). 2008;150:149-156.

16. Khoyratty F, Wilson $T$. The dentato-rubro-olivary tract: clinical dimension of this anatomical pathway. Case Rep Otolaryngol. 2013;2013:934386.

17. Gatlin JL, Wineman R, Schlakman B, Buciuc R, Khan M. Hypertrophic olivary degeneration after resection of a pontine cavernous malformation: a case report. J Radiol Case Rep. 2011;5:24-29.

18. Goyal M, Versnick E, Tuite P, et al. Hypertrophic olivary degeneration: metaanalysis of the temporal evolution of MR findings. AJNR Am J Neuroradiol. 2000;21:1073-1077.

19. Grueneisen JS, Ringelstein A, Forsting M. [Delayed but nonetheless characteristic: the clinical manifestation of hypertrophic degeneration of the olivary nucleus]. Rofo. 2013;185:881-883. German.

20. Shinohara Y, Kinoshita T, Kinoshita F, Kaminou T, Watanabe T, Ogawa T. Hypertrophic olivary degeneration after surgical resection of brain tumors. Acta Radiol. 2013;54:462-466. 
21. Yun JH, Ahn JS, Park JC, Kwon do H, Kwun BD, Kim CJ. Hypertrophic olivary degeneration following surgical resection or gamma knife radiosurgery of brainstem cavernous malformations: an 11-case series and a review of literature. Acta Neurochir (Wien). 2013;155: $469-476$.

22. Vyas S, Prabhakar S, Kumar A, Khandelwal N. Bilateral hypertrophic olivary degeneration. Ann Indian Acad Neurol. 2013;16:404-405.

23. Shinohara Y, Kinoshita T, Kinoshita F, Ogawa T. Hypertrophic olivary degeneration after gamma-knife radiosurgery for pontine metastasis. Magn Reson Med Sci. 2012;11:299-302.

24. Soria G, De Notaris M, Tudela R, et al. Improved assessment of ex vivo brainstem neuroanatomy with high-resolution MRI and DTI at 7 Tesla. Anat Rec (Hoboken). 2011;294:1035-1044.
25. Chen X, Weigel D, Ganslandt O, Buchfelder M, Nimsky C. Diffusion tensor imaging and white matter tractography in patients with brainstem lesions. Acta Neurochir (Wien). 2007;149:1117-1131.

26. Mori S, Zhang J. Principles of diffusion tensor imaging and its applications to basic neuroscience research. Neuron. 2006;51:527-539.

27. Phatouros CC, McConachie NS. Hypertrophic olivary degeneration: case report in a child. Pediatr Radiol. 1998;28:830-831.

28. Harter DH, Davis A. Hypertrophic olivary degeneration after resection of a pontine cavernoma. Case illustration. J Neurosurg. 2004;100:717.

\section{Publish your work in this journal}

Neuropsychiatric Disease and Treatment is an international, peerreviewed journal of clinical therapeutics and pharmacology focusing on concise rapid reporting of clinical or pre-clinical studies on a range of neuropsychiatric and neurological disorders. This journal is indexed on PubMed Central, the 'PsycINFO' database and CAS, and is the official journal of The International Neuropsychiatric Association (INA). The manuscript management system is completely online and includes a very quick and fair peer-review system, which is all easy to use. Visit http://www.dovepress.com/testimonials.php to read real quotes from published authors.

Submit your manuscript here: http://www.dovepress.com/neuropsychiatric-disease-and-treatment-journal 